\title{
Coexistence of Pernicious Anemia and Myasthenia Gravis Presenting As Dyspnea
}

\author{
Sara Khademolhosseini ${ }^{1}$, Elspeth Springsted ${ }^{2}$, Seyedmohammad Pourshahid ${ }^{3}$, Badri Giri ${ }^{3}$ \\ 1. Internal Medicine, Icahn School of Medicine at Mount Sinai, Queens Hospital Center, New York, USA 2. Internal \\ Medicine, Virginia Tech Carilion School of Medicine, Roanoke, USA 3. Pulmonary and Critical Care, Virginia Tech \\ Carilion School of Medicine, Roanoke, USA
}

Corresponding author: Seyedmohammad Pourshahid, omid.pourshahid@gmail.com

\begin{abstract}
Dyspnea is a common symptom and may be due to a multitude of conditions, including cardiopulmonary insufficiency, anemia, neuromuscular disorders, obesity, or deconditioning. It is not uncommon that more than one process contributes to shortness of breath. Here, we present a patient with a complaint of worsening shortness of breath who was found to have two very rare causes of dyspnea simultaneously. The symptoms resolved with treatment of pernicious anemia and myasthenia gravis (MG). The coexistence of pernicious anemia and MG is extremely rare, with only two other cases reported so far.
\end{abstract}

Categories: Internal Medicine, Pulmonology

Keywords: myasthenia gravis (mg), pernicious-anemia, dyspnea, multiple autoimmune diseases, coexistence

\section{Introduction}

Dyspnea, or subjective shortness of breath, is a common presenting symptom that affects millions of patients. With a wide range of differential diagnoses, dyspnea could be an early manifestation of pulmonary diseases, cardiovascular insufficiency, anemia, neuromuscular disorders, obesity, or deconditioning [1]. It is not uncommon that more than one process contributes to dyspnea. The providers must keep a broad differential diagnosis and investigate additional causes if the patient is not responding to treatment as expected. Although cardiopulmonary-associated shortness of breath constitutes a great proportion of cases, alternative etiologies need to be considered.

\section{Case Presentation}

A 73-year-old female with no significant medical history presented to the pulmonary clinic for dyspnea on exertion, gradually worsening over one year. She endorsed generalized weakness and frequent falls. She denied cough, fever, night sweats, or weight loss. On physical examination, she was afebrile with a heart rate of 81 beats/minute, blood pressure 141/86 $\mathrm{mmHg}$, respiratory rate of 16 breaths/minute. Oxygen saturation was $99 \%$ on room air. Lung sounds were clear to auscultation. Mild bilateral upper extremity weakness and ataxic gait were noted. Reflexes were brisk in the upper and lower extremities, and the Romberg test was positive.

Review began 05/24/2021 Review ended 05/26/2021 Published 05/28/2021

(c) Copyright 2021

Khademolhosseini et al. This is an open access article distributed under the terms of the Creative Commons Attribution License CC-BY 4.0., which permits unrestricted use, distribution, and reproduction in any medium, provided the original author and source are credited.

An extensive cardiac workup was nondiagnostic before referral to the pulmonary clinic, including echocardiography with preserved ejection fraction and normal stress test.

Pulmonary function test (PFT) (Figure 1) revealed a severe restrictive pattern and mildly reduced diffusing capacity of the lungs for carbon monoxide (DLCO). 


\section{Cureus}

\begin{tabular}{|c|c|c|c|c|c|c|c|}
\hline PFT & FEV1(L) & FVC(L) & FEV1/FVC & FEV1/SVC & TLC(L) & ERV(L) & $\begin{array}{c}\text { DLCO } \\
\text { (mL/min/mm Hg) }\end{array}$ \\
\hline Value & 1.69 & 2.13 & 79 & 74 & 2.40 & 0.12 & 15.05 \\
(Percent Predicted) & $(83 \%)$ & $(80 \%)$ & & & $(49 \%)$ & $(13 \%)$ & $(67 \%)$ \\
\hline
\end{tabular}

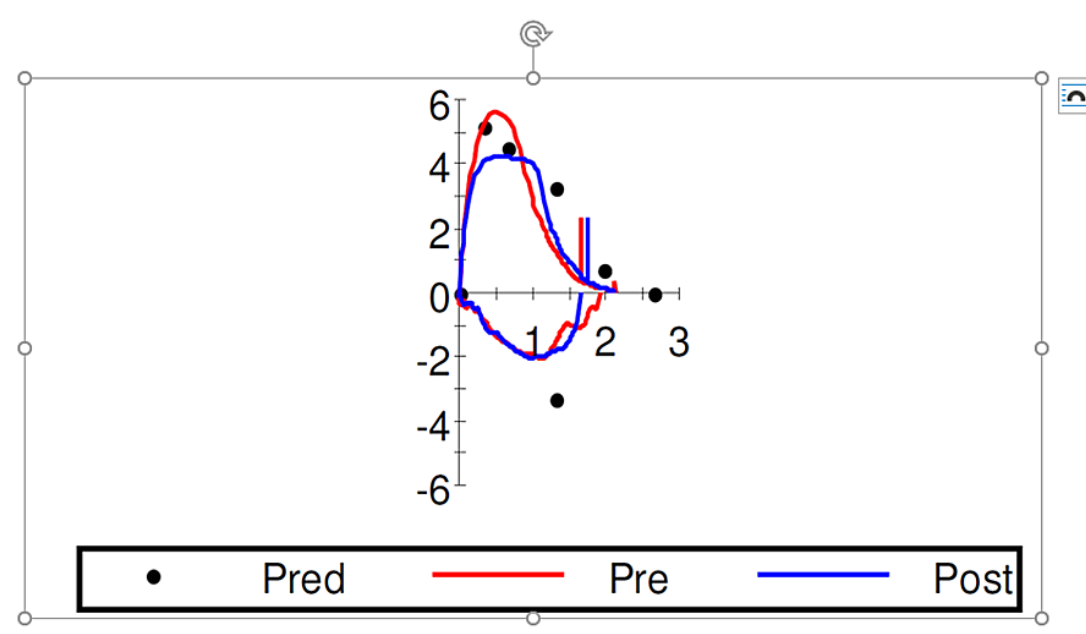

Figure 1. PFT and flow-volume loops

FIGURE 1: Pulmonary function test and flow-volume loops.

CT scan of the chest showed no evidence of parenchymal lung disease. The workup for connective tissue disease and hypersensitivity pneumonitis was unremarkable. The remarkable laboratory results are summarized in Table 1 .

\begin{tabular}{|c|c|c|}
\hline Remarkable lab & Value, unit & Interpretation \\
\hline Hemoglobin & $10.3 \mathrm{~g} / \mathrm{dl}$ & Decreased \\
\hline Mean Corpuscular Volume & $112 \mathrm{fL}$ & Elevated \\
\hline B12 & $<150 \mathrm{pg} / \mathrm{ml}$ & Undetectable \\
\hline Folate & $11.31 \mathrm{ng} / \mathrm{ml}$ & Normal \\
\hline Homocysteine & $102.7 \mathrm{umol} / \mathrm{L}$ & Elevated \\
\hline Methylmalonic Acid & $6450 \mathrm{nmol} / \mathrm{L}$ & Elevated \\
\hline Intrinsic Factor Block Ab & Positive & Abnormal \\
\hline Acetylcholine Receptor Block Ab & $27 \%$ inhibition & Increased inhibition \\
\hline Acetylcholine Receptor Bind Ab & $2.31 \mathrm{nmol} / \mathrm{L}$ & Elevated \\
\hline Acetylcholine Receptor Mod Ab & $76 \%$ inhibition & Increased inhibition \\
\hline
\end{tabular}

TABLE 1: Remarkable laboratory results.

Serology was positive for myasthenia gravis (MG). Electromyography and nerve conduction velocity showed a decrement in repetitive stimulation, but it was not detected until 10 minutes post-exercise. The patient was started on pyridostigmine, azathioprine, and prednisone to manage seropositive MG. Her MG medication doses were increased over three months, but her symptoms, especially dyspnea and generalized weakness, did not improve. Intravenous immunoglobulin therapy was started, and fortunately patient had a dramatic response with the first session. The patient reported relief from shortness of breath and weakness. 
She is currently co-managed by the neurology and pulmonology clinic.

\section{Discussion}

The PFT revealed a severely reduced total lung capacity (TLC), and mild reduction of DLCO and residual volume (RV) (DLCO was within normal limits when adjusted for alveolar ventilation). Forced expiratory volume (FEV1), forced vital capacity (FVC), and their ratio were normal. This pattern suggests a restrictive pattern with a wide range of differential diagnoses categorized into intrinsic lung diseases, the extrinsic disorders of the chest wall and pleura, and neuromuscular diseases [2]. There was no evidence of inherent lung diseases like lung parenchymal pathology or air space disease in chest CT that correlates with this degree of symptoms.

Usually, morbid obesity with BMI above 40 and neuromuscular diseases share some PFT changes such as reduced FEV1, FVC, expiratory reserve volume (ERV), and TLC, unchanged FEV1/FVC, and increased RV. Maximum inspiratory and expiratory pressure may be lower than the expected lower limit of normal for age and sex in patients with neuromuscular disorders [3]. In this case, BMI was only 31, and her extent of dyspnea did not correlate with obesity or deconditioning as she was getting short of breath while talking. No gross chest wall disease or pleural disorder was noted in the investigation.

Megaloblastic anemia could be contributing to her shortness of breath; however, her hemoglobin was stable and not low enough to explain her severe dyspnea.

B12 deficiency presents a wide range of features like macrocytic anemia, gastrointestinal symptoms, and a variable neurologic abnormality. Symmetric paresthesia and ataxic gait are the most common neurological findings [4]. Mood disorder, forgetfulness, and dementia are reported as well. Muscular weakness that may progress to paraplegia is seen with severe deficiency but is not a common finding [5].

Symptoms of tissue hypoxia may occur with severe anemia or the presence of underlying heart disease. Typically, anemia develops gradually, and the physiologic compensation mitigates symptoms of anemia like shortness of breath and palpitation [4]. Hence, dyspnea is rarely the presenting symptom of B12 deficiency, which could cause shortness of breath by a combination of symptomatic anemia and muscular weakness $[4,6,7]$. Pernicious anemia is a subtype of B12 deficiency caused by autoantibodies targeting intrinsic factors, interfering with the absorption of B12. Pernicious anemia is associated with other autoimmune conditions such as thyroid disease or vitiligo [7].

At this stage, alternative causes of dyspnea, such as neuromuscular disease was coming higher in the list of differentials. MG is an autoimmune disease that affects neuromuscular transmission. MG usually presents a combination of weakness in ocular, bulbar, skeletal, and respiratory muscles [8]. An anti-acetylcholine antibody has a specificity close to $99 \%$, which makes a false positive extremely unlikely. Also, the sensitivity of serology is $85 \%$ in generalized and close to $50 \%$ in ocular MG [9]. MG crisis, which causes respiratory failure, usually happens in the late stages of the disease, and patients have prior extensive neurological signs and symptoms. Isolated respiratory muscle weakness caused by MG is very rare and makes the diagnosis challenging [10]. MG is associated with other autoimmune diseases such as autoimmune thyroid disease or connective tissue disorders such as rheumatoid arthritis, lupus, and Sjogren's syndrome [11].

The coexistence of pernicious anemia and MG is extremely rare, and to the best of our knowledge, only two other cases have been reported so far [12,13].

\section{Conclusions}

Dyspnea is a widespread symptom with a broad differential diagnosis, including pulmonary diseases, cardiovascular insufficiency, anemia, neuromuscular disorders, obesity, or deconditioning. Providers must recognize the possibility of contribution from different etiologies. Cardiopulmonary-related dyspnea is one of the most prevalent, but other differentials like anemia and neuromuscular diseases need to be considered as well. In this case, the diagnosis of concurrent pernicious anemia and MG, which are rare causes of dyspnea, led to therapy with resolution of shortness of breath. The coexistence of pernicious anemia and MG is extremely rare, and to the best of our knowledge, only two other cases have been reported so far.

\section{Additional Information}

\section{Disclosures}

Human subjects: Consent was obtained or waived by all participants in this study. Conflicts of interest: In compliance with the ICMJE uniform disclosure form, all authors declare the following: Payment/services info: All authors have declared that no financial support was received from any organization for the submitted work. Financial relationships: All authors have declared that they have no financial relationships at present or within the previous three years with any organizations that might have an interest in the submitted work. Other relationships: All authors have declared that there are no other relationships or activities that could appear to have influenced the submitted work. 


\section{Cureus}

\section{References}

1. Parshall MB, Schwartzstein RM, Adams L, et al.: An official American Thoracic Society statement: update on the mechanisms, assessment, and management of dyspnea. Am J Respir Crit Care Med. 2012, 185:435-452. 10.1164/rccm.201111-2042ST

2. Boros PW, Enright PL, Quanjer PH, Borsboom GJ, Wesolowski SP, Hyatt RE: Impaired lung compliance and DL,CO but no restrictive ventilatory defect in sarcoidosis. Eur Respir J. 2010, 36:1315-1322.

10.1183/09031936.00166809

3. Chiang J, Mehta K, Amin R: Respiratory diagnostic tools in neuromuscular disease . Children (Basel). 2018, 5:78. 10.3390/children5060078

4. Green R: Vitamin B12 deficiency from the perspective of a practicing hematologist . Blood. 2017, 129:26032611. 10.1182/blood-2016-10-569186

5. Stabler SP: Vitamin B12 deficiency. N Engl J Med. 2013, 368:149-160. 10.1056/NEJMcp1113996

6. Bhattacharjee A, Easo Samuel A: Vitamin B12 deficiency in a patient presenting with dyspnea: a case report . Adv J Emerg Med. 2018, 3:e19. 10.22114/AJEM.v0i0.103

7. Mohamed M, Thio J, Thomas RS, Phillips J: Pernicious anaemia. BMJ. 2020, 369:m1319. 10.1136/bmj.m1319

8. Gilhus NE: Myasthenia gravis. N Engl J Med. 2016, 375:2570-2581. 10.1056/NEJMra1602678

9. Lazaridis K, Tzartos SJ: Autoantibody specificities in myasthenia gravis; implications for improved diagnostics and therapeutics. Front Immunol. 2020, 11:212. 10.3389/fimmu.2020.00212

10. Mandaliya R, Kulandaivel K, Nowotarski N, Buddhdev K, Patel R: A challenging diagnosis of fluctuating dyspnea: myasthenia gravis. J Clin Diagn Res. 2015, 9:OD06-OD08. 10.7860/JCDR/2015/12389.6015

11. Chang C-C, Lin T-M, Chang Y-S, Chen W-S, Sheu J-J, Chen Y-H, Chen J-H: Thymectomy in patients with myasthenia gravis increases the risk of autoimmune rheumatic diseases: a nationwide cohort study. Rheumatology (Oxford). 2019, 58:135-143. 10.1093/rheumatology/key236

12. Krol TC: Myasthenia gravis, pernicious anemia, and Hashimoto's thyroiditis. Arch Neurol. 1979, 36:594-595. 10.1001/archneur.1979.00500450088022

13. Chang K-H, Lyu R-K, Ro L-S, Wu Y-R, Chen C-M: Coexistence of pernicious anemia and myasthenia gravisa rare combination of autoimmune diseases in Taiwan. J Formos Med Assoc. 2006, 105:946-949.

10.1016/S0929-6646(09)60181-9 